\title{
THE IMPACT OF NUTRIENT LOADS ON THE BALTIC SEA.
}

\author{
Lars Rahm \\ Linkoping University, Sweden
}

The environmental quality of the Baltic Sea has been deteriorating for a long time, see e.g. the presently widespread hypoxic or anoxic bottoms. The bottom conditions affiect the ecosystem on various levels, one example is the Baltic cod population but also those biogeochemical processes that control the nutrient recycling are to a large extent controlled by the redox conditions. We can easily identify several areas of concern, pollution of toxic substances, loads of slowly degrading matters, loads of nutrients or other human derived loads. Focus here is on the nutrient complex. A common opinion is that the excessive loads of nutrients have lead to an eutrophication of this semi-enclosed brackish sea area. This has been of deep concern to the riparian countries of the Baltic; the forming of HELCOM (Helsinki Comission for the environment of the Baltic Sea) is just one such regional initiative. A great variety of processes on "all scales" are interacting to form this environment. Our knowledge about them and their interactions are limited, which is a problem for e.g. descision makers. Below is an attempt to present one possible way to gain some information of this system and that is by "robust" large scale analysis.

Simple nutrient budgets based on monitoring data have been formed describing the basic flows of this system. Continuity requirements make it possible to estimate the net fluxes and sources and sinks based on the hydrography of this system. A key to success is good input data, something that has improved considerably during the last decades. But major voids exist in our knowledge about the loads. For example, still there are no regular measures of the organic nitrogen load from the atmosphere. This despite reports indicating that this may reach about a half of the inorganic loads. Further, we don't know how much of this is bioavailable. The latter problem is also present in analysing the riverine data. How much of what enters the sea is bioavailable and on what time scales? Another uncertain load is nitrogen fixation, which is a rather special property of the Baltic Sea. Rough estimates suggest that it may be comparable with the atmospheric load but there are probably large interannual variations.

An ambition to increase the time resolution in these budgets to yearly or seasonal scales has, however, capsized due to lack of data. Further, previous problems with chemical analysis sets a definite lower boundary in time for these estimates. In fact, the only reliable observations we have on long time scales for the stoichiometric conditions of the Baltic are salinity, temperature, oxygen and maybe sight depth. For example, reliable nitrogen analysis has only been available during the last three decades. It is evident that the long adjustment time of this system will make 
it difficult to proper analyse its behaviour from the perspective of large climatic and hydrographic changes. The question is: are we really taking into account the essential loads and processes when we try to understand this transient system? Robust trend analysis describes the significant changes in nutrient concentrations in the pelagial since the seventies, but we are probably studying the details of this ecosystem after the big increase in nutrient load has occurred. There are rough calculations showing substantial changes in nitrogen and phosphorus load during this century, changes that are assumed to have occurred since the fifties. Are these changes significant? There are some comparative studies of benthos at different locations in the sea corroborating this.

The use of silica has been assumed to be a good eutrophication indicator for the aquatic environment of the northern hemisphere since it is assumed not to be influenced by human impact. The dissolved silica (DSi) concentration has been decreasing during the last three decades in the surface layers of the water column, which could be interpreted as a reasonable response on increased nutrient load and primary production (by diatoms). However, deep sediment cores taken from various places in the Baltic indicate that the burial rates have changed substantially in the past but that the beginning of this increase can be found in the middle of the nineteenth century. Not surprising this long-term increase may be interpreted as the ecosystem's answer on an increased human population and its corresponding agricultural and industrial activities. This is attributed the increased spring diatom blooms. In fact, $\mathrm{DSi} / \mathrm{N}$ ratios, from the same locations, seem to approach the "limiting Redfield ratio", indicating a possible transcendence to a silica limited system. Interesting as this may be, recent investigations show that silica is not that ideal indicator we assumed, but may be heavily affiected by river regulations. The ongoing rapid regulation of major rivers in the third world will probably render the same changes in silica load to their coastal zone as have been observed in the Baltic with probably substantial effects on the local food web and ecosystem.

The punch line is that in order to understand what is really going on "in the ongoing eutrophication of the Baltic Sea", we have to both widen our spatio-temporal perspective and to improve our understanding of what is really available of those nutrients supplied to this ecosystem and on which time scales this occur. 